\title{
COORDINACIÓN ENTRE LA MATERIA DE EDUCACIÓN FÍSICA Y LAS ACTIVIDADES FÍSICO-DEPORTIVAS EXTRAESCOLARES
}

\author{
Ma Dolores González Rivera \\ Universidad de Alcalá \\ Antonio Campos Izquierdo \\ Universidad Politécnica de Madrid
}

\begin{abstract}
RESUMEN: En este estudio se analiza la posible conexión de las clases de Educación Física con las actividades físico-deportivas extraescolares de los centros educativos de Educación Primaria de la Comunidad Autónoma de Madrid. La investigación se enmarca dentro de la metodología de tipo cuantitativo de corte descriptivo, a través de encuesta, en la cual se ha utilizado como instrumento de recogida de datos la entrevista estandarizada por medio de cuestionario creado ad hoc, que fue completado por 300 docentes. En el estudio se obtiene que la gran mayoría del profesorado de las actividades físico-deportivas extraescolares aseguran no establecer ninguna coordinación con el profesorado de Educación Física del centro educativo. Sin embargo, la mayoría de estos docentes consideran que sería positivo que se produjera una coordinación con el profesorado de Educación Física. No obstante, más de la mitad de los docentes de las actividades físico-deportivas extraescolares opinan que los objetivos de las actividades que imparten no guardan relación con los objetivos del área de Educación Física.
\end{abstract}

ABSTRACT: In this article there is analyzed the possible connection of physical education classes with the extracurricular physical activities in the Primary School of the Region of Madrid. This research places inside the methodology of quantitative type of descriptive cut, across survey, which has been in use as instrument of withdrawal of information the interview standardized by means of questionnaire created ad hoc, that was completed by 300 teachers. In the study there is obtained that the vast majority of the teachers who give classes in the extracurricular physical activities say they do not establish any coordination with the Physical Education teacher of the school. However, most of these teachers feel they would be good if there were a coordination with the teachers of Physical Education. However, more than half of teachers who give classes in the extracurricular physical activities believe that 
the objectives of the activities they provide are not related to the objectives of the Physical Education.

PALABRAS CLAVE: Profesorado, actividad extraescolar, coordinación, Educación Física.

KEYWORDS: Teachers, extracurricular activities, coordination, Physical Education.

Recibido: $24 / 04 / 2010$

Aceptado: 23/02/2011

\section{INTRODUCCIÓN}

Ante la grave crisis de valores, de violencia, de aislamiento, de sedentarismo, de baja autoestima y de obesidad que sufren gran parte de los escolares en nuestra sociedad actual, la adecuada utilización del tiempo de ocio se ha convertido en una necesidad social (Santos, 1998).

Ante esta realidad social, el centro educativo, como institución cultural y productora de cultura, debe formar parte abriendo sus puertas y extendiendo sus horarios a otros espacios de vital importancia en la formación de los niños y niñas, con la finalidad de adquirir hábitos de vida saludables y de favorecer la creación de espacios de socialización y de integración (Macarro, 2002; Rivadeneyra, 2003 y Luengo, 2007).

Por tanto, el centro educativo constituye un espacio idóneo para la promoción y desarrollo de la práctica de actividades físicas y deportivas debido a su carácter formativo, ya que, si se promociona el deporte escolar en los centros educativos, se favorecerá la conexión y complementación de estas actividades con las intenciones del período lectivo, la educación de los jóvenes para que adquieran los valores sociales inherentes a la práctica deportiva, hábitos y actitudes saludables en la ocupación de su tiempo de ocio, la disminución de posibles problemas de salud y la continuidad en la realización de la actividad física y deporte durante su vida (Sánchez y Sánchez, 2003; Montesinos, 2005 y Orts, 2005).

Al respecto, en el manifiesto sobre el deporte en edad escolar de la Federación Española de Municipios y Provincias (1992), se resalta la importancia del centro escolar en el desarrollo de las prácticas deportivas en general, puesto que se manifiesta que éste constituye una de las piedras angulares en su organización. Por tanto, este espacio desempeña un papel importante en el desarrollo de las actividades físicas y deportivas, y, junto con la familia (porque es la primera unidad de socialización del niño/a), debe contribuir a la formación físico-deportiva básica que le traslade al niño/a a la iniciación frecuente de la práctica deportiva.

Son numerosos los autores que manifiestan la importancia de la presencia de la actividad física y el deporte en el centro educativo en horario no lectivo. Así, Delgado (2002) expone que, de entre los diferentes ámbitos de práctica del deporte en la etapa escolar, es el centro educativo donde mejor se puede garantizar un deporte educativo y formativo, adoptando un modelo de deporte en edad escolar participativo, polideportivo, coeducativo e integrador. Además, este autor añade que la mejora de la calidad de la enseñanza se corresponde con la capacidad de los centros educativos para ampliar su intervención educativa fuera del horario lectivo, disponiendo, al ser- 
vicio del conjunto de la comunidad escolar, de todos sus recursos materiales y humanos para el desarrollo de las actividades, entre otras, las de carácter físico-deportivo.

Igualmente, Fraile y De Diego (2006) manifiestan que desde hace tiempo el deporte escolar es considerado como una actividad de gran relevancia para el ámbito educativo, así como un excelente medio para intervenir en diversos problemas de nuestro entorno educativo y social.

En este sentido, González, Blasco, y Campos (2005) señalan que toda actividad físico-deportiva que se desarrolle en edad escolar debe enfocarse desde una perspectiva claramente educativa, tanto las que se impartan en instituciones fuera del contexto escolar como dentro de éste, pero aún más si cabe, en estas últimas, dado que estamos hablando de actividades dentro de un marco escolar.

Por todo ello, Montesinos (2005) reivindica como punto esencial en el deporte escolar el reconocimiento de los centros educativos como un espacio de intervención preferente para la promoción de la práctica de actividades físicas y deportivas así como el papel del profesorado de Educación Física como inductor de esta práctica entre los escolares. También, Álamo, Amador y Pintor (2002) exponen que, para que exista un modelo escolar educativo y formativo, es imprescindible que los entrenadores del deporte escolar se encuentren estrechamente vinculados al ámbito educativo y de manera específica, coordinados con el profesorado de Educación Física.

A partir de estas consideraciones, se pone de manifiesto la gran conexión que posee la actividad físico-deportiva con la materia de Educación Física, ya que aunque ésta, a diferencia de las actividades físico-deportivas extraescolares, tiene carácter obligatorio y formal, ambas se desarrollan en una misma etapa escolar, ambas son realizadas por los escolares y las dos benefician la formación integral del alumnado, favoreciendo su desarrollo físico, intelectual, emocional y social a partir de un modelo educativo global.

Así, Piéron (2001) manifiesta que el alumnado, durante el tiempo que pasa en la escuela no alcanza un volumen de actividad a un nivel apropiado que permita efectos significativos para la mejora de la salud o para actuar como una medida para la prevención de factores de riesgo, ya que tal y como apuntan González y Otero (2005) a pesar de que la Educación Física es obligatoria dentro del sistema educativo de seis a diecisiete años, es escasa su carga horaria, debido a que tan sólo constituye de una a dos horas semanales y, en ocasiones el tiempo dedicado a realizar actividad física y deporte durante las clases de forma vigorosa no alcanza los diez minutos.

Es tal la importancia de la existencia de una conexión de las clases de Educación Física y las actividades físico-deportivas extraescolares, que en el estudio de Moreno, Rodríguez y Gutiérrez (1997) se observó, en el alumnado con edades comprendidas entre 12 y 15 años, que cuanto mayor era el número de sesiones de Educación Física (en comparación con el alumnado que recibía menos sesiones de Educación Física), más convencidos estaban de la utilidad de la Educación Física para su futuro y más preferían la práctica de actividades físicas frente a otras actividades. Igualmente, Mollá (2007) en su estudio sobre la influencia de las actividades extraescolares y complementarias ofrecidas desde la asignatura de Educación Física en alumnado de cuarto de la ESO en el municipio de Alcoben- 
das, obtiene que un $88 \%$ del alumnado que participa en estas actividades manifiesta que sus experiencias han sido buenas o muy buenas y para el $49 \%$ la participación en estas actividades les sirvió para introducirles e interesarles en alguna actividad física-deportiva. Además, en el estudio de Torre (1997) se concluyó que las experiencias vividas en las clases de Educación Física son muy significativas a la hora de elegir si desean participar en las actividades extraescolares, de tal manera que existen mayores posibilidades para que se involucren en la práctica deportiva fuera del horario lectivo, cuanto mayores sean sus experiencias y percepciones en el horario lectivo a través de las clases de Educación Física.

Sin embargo, y a pesar de que la actividad físico-deportiva extraescolar se viene cubriendo como prolongación de la tarea educativa dentro de los centros educativos, en la mayoría de los casos se realiza de una forma no muy adecuada, ya que se aleja de su contexto educativo y formativo (Salvador, 1998). A esta consideración, se suman autores como Macazaga (2004), González y Otero (2005) y Tani (2007) que ponen de relieve el escaso éxito del área de conocimiento de la Educación Física en la promoción de actividades físicas y deportivas fuera del horario escolar y creen que es debido a la escasa conexión con las actividades deportivas extraescolares.

Igualmente, es necesaria esta conexión para que los objetivos, la adquisición de las competencias básicas, criterios de evaluación y metodología utilizada vayan en la misma dirección, más aún cuando diferentes autores manifiestan la posible deficiente intervención didáctica de las personas que trabajan en las actividades físicodeportivas extraescolares (González Rivera y Campos Izquierdo, 2010; Mitchell, Oslin y Griffin, 2006; Petry, Froberg y Madella, 2008).

Por tanto, es imprescindible la presencia del profesorado de Educación Física en las actividades físico-deportivas donde se desarrollan estas actividades en las tareas de coordinación y de supervisión en las actividades físico-deportivas extraescolares para favorecer su orientación educativa (Giménez, 2000). En esta línea, Gómez (1997) señala que estos profesionales, dado los conocimientos de educación físico-deportiva, deben ser los encargados de la integración del deporte escolar en la educación del alumnado, junto con otros agentes de la comunidad educativa.

Sin embargo, esta falta de implicación por parte del profesorado de Educación Física en las actividades físico-deportivas extraescolares que se desarrollan en su centro educativo, se puede deber a la falta del reconocimiento de esta labor por el sistema educativo. De esta forma, Álamo (2004) expone que la administración educativa debería esforzarse más en propiciar la implicación del profesorado en estas actividades en las tareas de coordinación, a través de una reducción de la carga lectiva o por medio de algún tipo de compensación económica. Por su parte, Ureña (2000) añade que se podría recoger en los horarios del profesorado de Educación Física horas lectivas para la organización y el diseño de las actividades físico-deportivas extraescolares.

Por tanto, ante el contexto descrito, en este estudio se analiza la existencia de la conexión de las clases de Educación Física con las actividades físico-deportivas 
extraescolares de los centros educativos de Educación Primaria de la Comunidad Autónoma de Madrid. Los objetivos de este estudio son los siguientes:

- Conocer la relación del profesorado de las actividades físico-deportivas con el profesorado de Educación Física del centro educativo y sus motivos.

- Indagar sobre la consideración de la necesidad de esta posible coordinación entre los profesores de las actividades físico-deportivas extraescolares y los profesores de la materia de Educación física.

- Analizar la relación entre los objetivos de las actividades físico-deportivas extraescolares con los objetivos de la materia de Educación Física.

\section{Método}

La metodología que se ha seguido en esta investigación ha sido cuantitativa de corte descriptivo. En el desarrollo de esta metodología, los procedimientos que se han seguido son los propios de una encuesta seccional, aplicada a una muestra de personas que trabajan en la función de docencia de las actividades físico-deportivas extraescolares en los centros educativos de Educación Primaria en la Comunidad Autónoma de Madrid. La encuesta se ha realizado mediante la realización de entrevista personal estandarizada por medio de cuestionario en los centros educativos a cada una de las personas seleccionadas de una muestra estadísticamente representativa.

Para establecer el universo objeto de estudio (número de docentes de actividades físico-deportivas extraescolares en los centros educativos de Educación Primaria) se ha seguido los estudios de Martínez del Castillo (1992) y de Campos Izquierdo (2007) ya que, como explica Heinemann (2003), los puestos de trabajo referidos a este mercado laboral no se recogen estadísticamente de forma específica y por ello es difícil conocer exactamente su cuantificación, por lo que han de deducirse de diversos cálculos, encuestas y estadísticas. El universo que se obtuvo fue de 1.100 personas que trabajan en la función de docencia de las actividades físicodeportivas extraescolares en los centros educativos de Educación Primaria en la Comunidad Autónoma de Madrid.

\section{Muestra}

El tamaño de la muestra real fue de 300 personas que desarrollan la función de docencia de las actividades físico-deportivas extraescolares en los centros educativos de Educación Primaria en la Comunidad Autónoma de Madrid. Dado una población de 1.100 personas, partiendo de los estudios de Martínez el Castillo (1992) y Campos Izquierdo (2007), y trabajando con un intervalo de confianza del 95,5\%, y suponiendo en la varianza poblacional el caso más desfavorable de p igual a $50 \%$, luego $q=50 \%$, el margen de error permitido de muestreo es de $\pm 4,93 \%$.

La afijación de la muestra ha sido proporcional a la distribución de las personas según tamaño demográfico de los municipios de la Comunidad de Madrid y según la situación geográfica respecto a las cinco áreas territoriales de esta Comunidad Autónoma. Además, se realizaron diversos submuestreos teniendo como objetivo 
dispersar la muestra para que influyera positivamente en la precisión de las estimaciones. El tipo de muestreo, ha sido probabilístico de tipo polietápico, estratificado en primera fase, por conglomerados (Bryman, 2004), siendo las unidades de primera etapa las áreas geográficas en que desarrollan los docentes su trabajo. El resto de unidades de etapa han sido en el siguiente orden hasta llegar a las unidades últimas, es decir los docentes de actividades físico-deportivas extraescolares en los centros educativos de Educación Primaria a entrevistar, las siguientes (dentro de cada área seleccionada): municipio y centro educativo, donde se localizó, entrevistó e identificó a la persona objeto de estudio.

\section{Material}

Para captar la información necesaria para los objetivos del estudio, se decidió partir del "Cuestionario para el estudio de la intervención docente y recursos humanos en el deporte en edad escolar" (González, 2007). Además, en cuanto a la validez de contenido fue validado por ocho especialistas externos al equipo de investigación. En el pretest, el instrumento fue administrado a cincuenta personas pertenecientes al universo estudiado. Todos los sujetos entendieron las cuestiones y alternativas de respuesta, y no hubo valores perdidos. Este instrumento de recogida de datos posee una fiabilidad de consistencia, lo que le da una estabilidad, ya que se ha utilizado posteriormente en diferentes investigaciones como las de González Rivera (2008, 2010) y Hernández (2008).

La entrevista estructurada por medio de cuestionario se compone de 55 preguntas cerradas que recogen cinco dimensiones: características sociodemográficas de las personas que trabajan en estas actividades (dimensión 1), características estructurales y organizativas de las actividades físico-deportivas extraescolares (dimensión 3), aspectos didácticos de las personas que imparten las actividades físico-deportivas extraescolares (dimensión 3), características laborales (dimensión 4) y características formativas. De este cuestionario, para el desarrollo del presente estudio, ha sido seleccionada la tercera dimensión, cuyas variables analizadas son: relación con la comunidad educativa y objetivos de las actividades y los ítems relacionados con los objetivos del presente estudio: relación con el profesorado de Educación Física (ítem 18), valoración de la relación con el profesorado de Educación Física (ítem 19), relación con los objetivos de Educación Física (ítem 20) y motivos de la ausencia de relación con los objetivos de Educación Física (ítem 21). En la tabla 1 se exponen las preguntas del cuestionario seleccionadas para este estudio. 
Tabla 1. Preguntas del cuestionario seleccionadas para el estudio

18. ¿Mantiene algún tipo de relación sobre la actividad con el profesorado de Educación física del centro en el cual imparte las actividades? (se admiten varias respuestas)

No, no tiene ningún tipo de relación con él/ella.

Sí, para mejorar los aspectos organizativos

Sí, están coordinados en la programación de las actividades

Sí, para mejorar los aspectos docentes y físico-deportivos

Otros

19. ¿Piensa que es positiva y/o necesaria una coordinación con el profesorado de Educación Física del centro para la mejora de las actividades extraescolares?

Sí, piensa que es positiva y necesaria

Sí, piensa que es positiva pero no necesaria

No, piensa que ni es positiva ni necesaria

$\mathrm{Ns} / \mathrm{nc}$

20. ¿Tienen relación los objetivos de la/s actividad/es extraescolar/es que imparte con los del área de Educación Física?

Sí.....

No.

En algunas actividades sí y en otras no

Ns/nc.

21. (Dirigida sólo a quien no haya respondido de forma afirmativa a la pregunta anterior) ¿Por qué piensa que no tiene relación los objetivos de la/s actividad/es extraescolar/es que imparte con los del área de Educación Física? (se admiten varias respuestas)

Porque los objetivos del área de E.F, no se adecuan a los de las actividades extraescolares.

Porque desconoce los objetivos del área de E.F.

Otros

$\mathrm{Ns} / \mathrm{nc}$

1
2
3
4
Por

1
2
3
4

\section{Procedimiento}

La investigación ha sido de corte transversal, debido a que la recogida de la información se llevó a cabo entre los meses de octubre de 2006 a mayo de 2007. Toda la fase de procedimiento de recogida de la información fue realizada por un único entrevistador, con la finalidad de obtener una mayor fiabilidad y validez en el estudio (García Ferrando, 2002). Las entrevistas se realizaron de forma personal e individual cara a cara a cada uno de los individuos seleccionados de la muestra en los centros educativos y tuvieron una duración promedio de quince minutos.

El análisis de datos ha sido efectuado, tras ser tabulados y mecanizados los datos informáticamente. Se ha realizado un análisis descriptivo univariable y bivariable, empleando el paquete de programas SPSS para WINDOWS (V 14.0).

\section{RESULTADOS}

El $76,3 \%$ del profesorado afirma que no mantiene ningún tipo de relación, respecto a las actividades extraescolares, con el profesorado de Educación Física del centro educativo donde imparte dichas actividades. Sólo un 23,7\% mantiene algún tipo de coordinación con el profesorado de Educación Física (gráfico 1). 
Gráfico 1. Relación con el profesorado de Educación Física del centro educativo

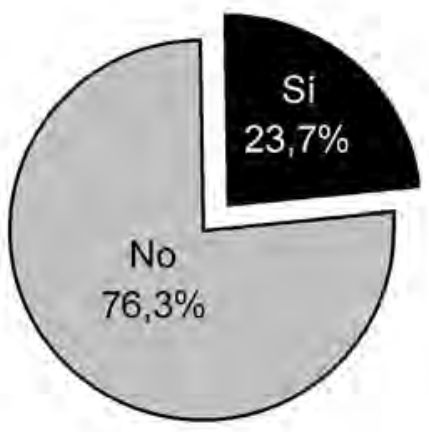

Tal y como se observa en el gráfico 2, las personas que imparten las actividades físico-deportivas extraescolares en los centros educativos de titularidad privada se coordinan con el profesorado de Educación Física del mismo centro educativo en mayor medida que los de titularidad pública, con un porcentaje del $40 \%$ del total de las personas, frente a un $12 \%$ en los centros públicos.

Gráfico 2. Relación con el profesorado de Educación Física del centro educativo en función de la titularidad del centro

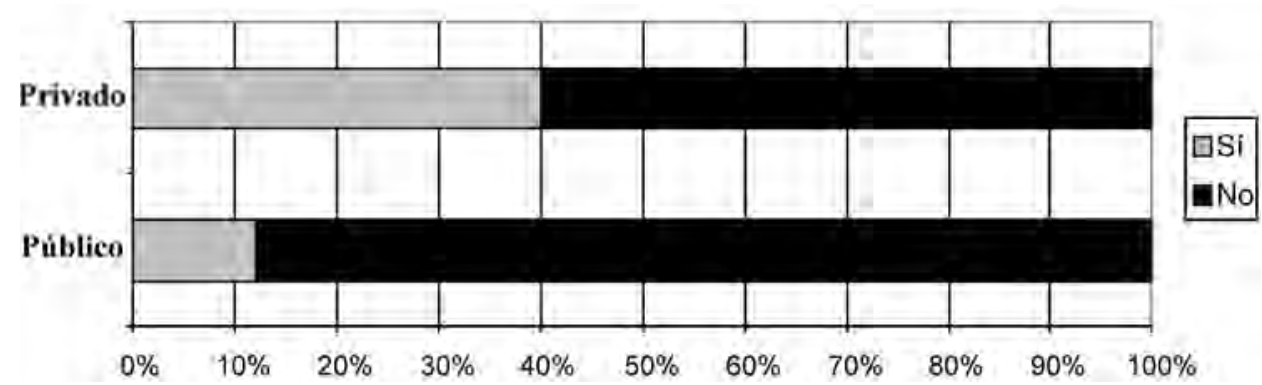

Los motivos por los que el profesorado de actividades físico-deportivas extraescolares se relaciona con el profesorado de Educación Física del centro educativo son varios (ver gráfico 3): un 40\% afirma que mantiene dicha relación para mejorar aspectos organizativos, un $27 \%$ para mejorar aspectos tanto docentes como físicodeportivos del alumnado, un $21 \%$ se debe a que el profesor o la profesora de Educación Física es el/la coordinador/a de las actividades extraescolares y, por último, un $12 \%$ responde que ellos/as mismos/as son los profesores/as de Educación Física del centro donde imparten las actividades. 
Gráfico 3. Motivos por los que se relaciona con el profesorado de Educación Física del centro educativo

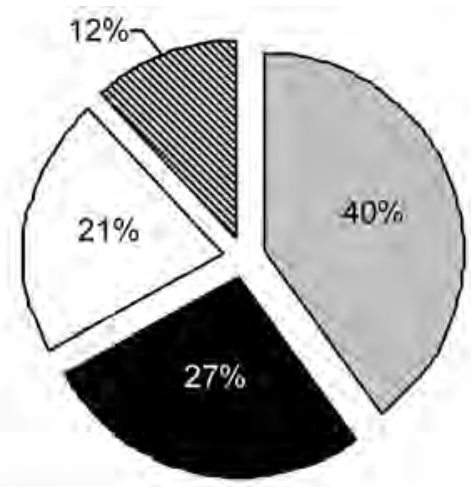

GAspectos organizativos

E EI/la profesor/a de E.F. es el coordinador/a
Aspectos docentes y físico-deportivos

E. Élella es profesor/a E.F. del centro

De todos estos motivos, el hecho de relacionarse con el profesorado de Educación Física con la finalidad de mejorar los aspectos organizativos es mayoritario en los centros educativos de titularidad pública (con un $83 \%$ del total de los motivos en este tipo de centros), el resto de las causas se presentan en mayores porcentajes en los centros privados, hasta tal punto de que el hecho de que el/la profesor/a de Educación Física es el/la coordinador/a de las actividades físico-deportivas extraescolares es sólo propio de estos centros y presentan un elevado porcentaje sobre los centros públicos debido a que el profesorado de Educación Física también lo es de las actividades físico-deportivas extraescolares, y, en algunos casos, son obligados por el centro privado en el que trabaja (ver gráfico 4).

Gráfico 4. Motivos por los que se relaciona con el profesorado de Educación Física del centro educativo según la titularidad del centro educativo

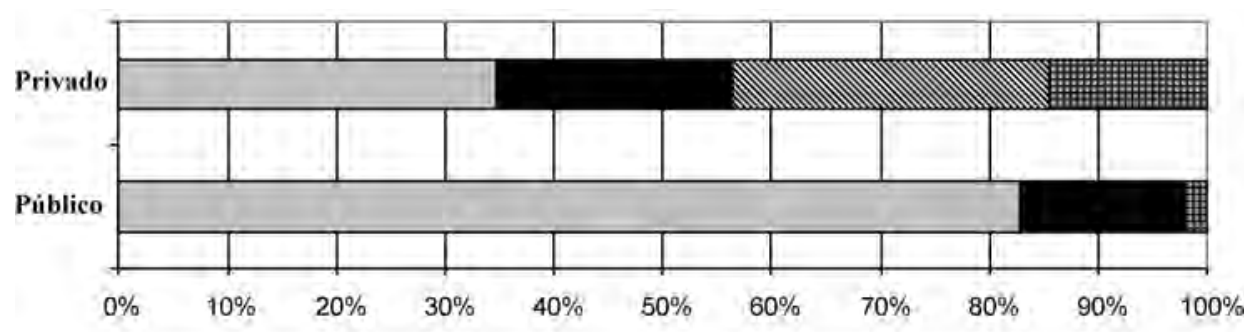


A pesar del bajo porcentaje que existe en cuanto a una coordinación con el profesorado de Educación Física (ver gráfico 5), la mayoría de las personas que imparten las actividades físico-deportivas extraescolares (el 66\%) considera que es o sería positivo que se produzca o produjera esta coordinación, de las que un 36,3\% de las personas opina que es o sería positiva y necesaria, sin embargo, un $29,7 \%$ de las mismas manifiesta que no es o sería necesaria una coordinación (a pesar de que es o sería positiva). Un 32,7\% de las personas responde que ni es positiva ni necesaria una coordinación con el profesorado de Educación Física del centro educativo donde imparten las actividades.

Gráfico 5. Consideración de la necesidad de coordinación con el profesorado de Educación Física del centro educativo

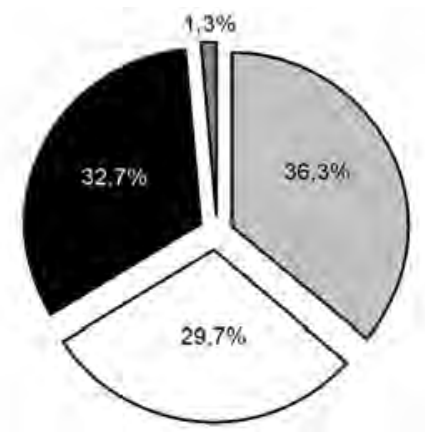

Si, positiva y necesaria

Ni positiva ni necesaria
Si positiva y no necesaria

$\square$ Ns/ne

En cuanto a los objetivos de las actividades físico-deportivas extraescolares y su relación con los del área de conocimiento de Educación Física, en el gráfico 6 se observa que más de la mitad del profesorado que imparte las primeras (un 58,3\%) manifiesta que sus objetivos no tienen ni deben tener ninguna relación con los objetivos del área de Educación Física, en cambio, un 41,7\% afirma que sí existe relación entre ambos objetivos.

Gráfico 6. Relación entre los objetivos de las actividades físico-deportivas extraescolares con los objetivos del área de conocimiento de Educación Física

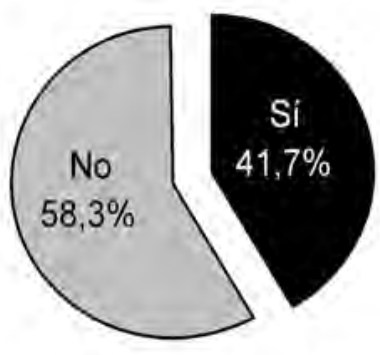


De entre el profesorado cuyos objetivos no tienen ninguna relación con los del área de conocimiento de Educación Física (ver gráfico 7), los motivos por los que es así son: porque no se adecuan los objetivos de las actividades físico-deportivas extraescolares que imparte con los de la asignatura de Educación Física (el 50,3\% de este profesorado), porque desconoce los objetivos de Educación Física (el $46,4 \%$ ) y porque es el profesor de Educación Física quien se debe preocupar de la posible relación entre los objetivos de ambos (3,3\%).

Gráfico 7. Motivos por los que se considera que los objetivos de las actividades físico-deportivas no tienen relación con los de Educación Física

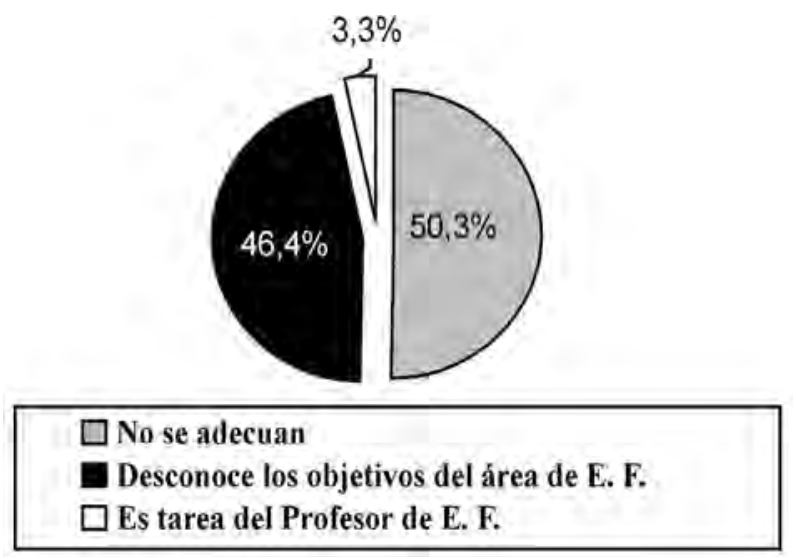

\section{Discusıón}

En este estudio se obtiene que la gran mayoría del profesorado de las actividades físico-deportivas extraescolares (el 73,3\%) asegura no establecer ninguna coordinación con el profesorado de Educación Física del centro educativo, y tan sólo el 5,6\% del profesorado afirma que el maestro/a de Educación Física es el coordinador de las actividades físico-deportivas (este aspecto es exclusivo de los centros educativos privados). Este hecho coincide con la afirmación del informe del Parlamento Europeo (2007), ya que sostiene que no parece existir una coordinación adecuada orientada a conciliar las actividades físico-deportivas escolares y extraescolares. Por su parte, Orts (2005) expone que la habitual desconexión de la asignatura de Educación Física con las actividades físico-deportivas extraescolares provoca desajustes entre los valores educativos y deportivos que se transmiten en la escuela con lo que el alumnado aprende en estas actividades (necesidad de ganar, prestigio, rivalidad, ejercicios contraproducentes para la salud etc.). Por tanto, ante esta situación y para facilitar dicha conexión, el profesorado de Educación Física de los centros educativos donde se imparten las actividades físico-deportivas extraescolares y las personas que imparten estas actividades extraescolares de los mismos centros educativos deben estar coordinados, sobretodo en las etapas de Educación Infantil y Primaria, ya que el profesorado de Educación Física puede ser el gran impulsor de un enfo- 
que participativo y educativo de estas actividades. En este sentido, Gómez (1997), Giménez (2000), Álamo (2000) y Ortúzar (2005) defienden la imprescindible presencia del profesorado de Educación Física en las tareas de coordinación en las actividades físico-deportivas extraescolares para favorecer su orientación educativa.

Además, en el estudio se obtiene que del escaso porcentaje del profesorado de las actividades físico-deportivas extraescolares $(23,7 \%)$ que mantiene relación con el maestro/a de Educación Física, el de los centros educativos de titularidad privada se relaciona en mayor medida que el profesorado de los centros de titularidad pública. Estos hechos coinciden con las consideraciones de Velázquez, Devís, De la Torre, Carranza y Fraile (2004) ya que exponen que la implicación del profesorado de Educación Física en las actividades físico-deportivas en la mayoría de los casos es inexistente, agravándose en caso de los centros públicos. Igualmente, en el estudio de Díaz (2005) se obtiene que la implicación de los/as maestros/as de Educación Física de los centros educativos públicos en las actividades físico-deportivas extraescolares es mínima, mientras que en los centros privados es mayor. Ahora bien, se debe señalar que los centros educativos de titularidad privada obligan, de alguna manera, a su profesorado de Educación Física a realizar tareas de coordinación u organización en las actividades extraescolares, y, por otra parte, la administración educativa no reconoce ni propicia la implicación del profesorado de los centros públicos en las tareas de coordinación de las actividades extraescolares. En esta línea, autores como Álamo (2004) y Ureña (2000) señalan la importancia de este reconocimiento por parte de las autoridades educativas, bien mediante una compensación económica, bien mediante la reducción de la carga lectiva, estableciendo horas lectivas destinadas a la organización y diseño de las actividades físico-deportivas extraescolares. Por otra parte, Ortúzar (2005), en su estudio, expresa la reticencia que tiene el profesorado de Educación Física, especialmente el perteneciente a los centros educativos de titularidad pública, en la implicación en estas actividades, a pesar de que este colectivo reconoce la importancia de la figura del profesorado en las mismas. De hecho, Flintoff (2003), manifiesta que la creación de programas de coordinación entre la Educación Física y el deporte extraescolar con agentes deportivos de la comunidad local (tales como el programa deporte-escuela que se está desarroIlando en Inglaterra y Gales), está creando tensiones en el profesorado de Educación Física.

Los motivos por los que el profesorado de actividades físico-deportivas extraescolares se relaciona con el profesorado de Educación Física del centro educativo son, entre otros, para mejorar aspectos organizativos (un 40\%) y tan sólo un $27 \%$ para mejorar aspectos tanto docentes como físico-deportivos del alumnado. Este último aspecto debería ser tenido más en cuenta a la hora de establecer las líneas de coordinación, ya que debería existir una adecuada coordinación para que se compartieran los mismos objetivos, contenidos y estrategias metodológicas (Velázquez et al. 2004). Además, esta necesaria coordinación es imprescindible ya que diversos estudios demuestran la falta de formación por parte de las personas que trabajan en la función de docencia con alumnado en edad escolar (Augustin, 2003; Camy, Chantelat y Le Roux, 1999; González y Campos, 2010; Parker, 2000; Shibli, Taylor, Nichols, Gratton, y Kokolakakis, 1999). En este sentido, Campos Izquierdo y 
Tomás (2005) exponen como una de las principales líneas de actuación de los Juegos Deportivos Municipales de la ciudad de Valencia, la colaboración con el profesorado de Educación Física de los centros educativos con el fin de dotar de una mayor orientación educativa a las actividades físico-deportivas extraescolares, e incluso en su orientación competitiva.

A pesar del bajo porcentaje que existe en cuanto a una coordinación con el profesorado de Educación Física, la mayoría de las personas que imparten las actividades físico-deportivas extraescolares (el 66\%) considera que es o sería positivo que se produzca o produjera esta coordinación. Al respecto, diversos autores (Giménez, 2000; Feu, 2002) afirman la necesidad de una oferta amplia, variada y adecuada de la actividad física y deportiva en edad escolar que complemente la Educación Física integrada en el sistema educativo y, más aún cuando diferentes estudios demuestran que el horario lectivo de esta área de conocimiento no es suficiente, tanto en la frecuencia como en la duración, para alcanzar los objetivos y los beneficios de la actividad física y el deporte. Por tanto, las actividades físico-deportivas extraescolares se convierten en un complemento del área de conocimiento de Educación Física, es decir, en un instrumento más al servicio del profesorado de Educación Física, favoreciendo la promoción de la actividad físico-deportiva en un ambiente menos formal y más distendido, con un carácter más recreativo con la finalidad de motivar hacia la práctica de la actividad física y el deporte en edades adultas (Mollá, 2007).

Más de la mitad de los docentes de las actividades físico-deportivas extraescolares de este estudio (el 58,3\%) opinan que los objetivos de las actividades que imparte no guardan relación con los objetivos del área de Educación Física de Educación Primaria. Ello se debe a que la mitad del profesorado piensa que los objetivos de estas actividades no se adecuan a los de la Educación Física, y casi la otra mitad del mismo afirma que se debe a que desconoce los objetivos de esta área de conocimiento. Este aspecto coincide con las afirmaciones de Martín, Cacho y Martín (2001) que exponen que los objetivos del área de Educación Física como el de las actividades físico-deportivas extraescolares no están en consonancia y, en muchos casos estos objetivos divergen. Así, la actividad física y deportiva extraescolar está ligada teóricamente a complementar la Educación Física, pero en la realidad éstas carecen de un objetivo académico. Por tanto, ante esta realidad, es imprescindible la presencia del profesorado de Educación Física en las actividades físico-deportivas donde se desarrollan estas actividades en las tareas de coordinación y de supervisión en las actividades físico-deportivas extraescolares para favorecer su orientación educativa a partir de la interrelación de los objetivos que permitan alcanzar los fines de la educación (Giménez, 2000). En esta línea, Gómez (1997) señala que estos profesionales, dado los conocimientos de educación físico-deportiva, deben ser los agentes encargados de la integración del deporte escolar en la educación del alumnado. De esta forma se conseguirá una mayor orientación educativa en estas actividades, dada la inadecuada intervención didáctica que parece ser que se produce debido a una insuficiente formación de los docentes del deporte escolar (Campos Izquierdo, 2007; Griffin y Butler, 2005). 


\section{CONCLUSIONES}

La gran mayoría del profesorado de las actividades físico-deportivas extraescolares asegura no establecer ningún tipo de relación con el profesorado de Educación Física del centro educativo, si bien el profesorado de los centros educativos privados se relaciona con los docentes del área de Educación Física en mayor medida que el profesorado de los centros públicos. También se obtiene que un bajo porcentaje del profesorado expone que el motivo por los que el profesorado de las actividades físico-deportivas extraescolares se relaciona con el profesorado de Educación Física del centro educativo es para mejorar aspectos tanto docentes como físico-deportivos del alumnado. Sin embargo, la mayoría de las personas que imparten las actividades físico-deportivas extraescolares considera que sería positivo que se produjera una coordinación con el profesorado de Educación Física. No obstante, más de la mitad de los docentes de las actividades físico-deportivas extraescolares opinan que los objetivos de las actividades que imparte no guardan relación con los objetivos del área de Educación Física de Educación Primaria. Por tanto, se observa que no existe una adecuada conexión entre las actividades físico-deportivas y las clases de Educación Física, por lo que para favorecer una adecuada educación físico-deportiva se deben establecer unas líneas de actuación de mejora en la organización y desarrollo de las actividades físico-deportivas extraescolares que permitan una verdadera complementariedad.

\section{REFERENCIAS BIBLIOGRÁFICAS}

ÁlAMO, J. M. (2000). Análisis del deporte escolar en la isla de Gran Canaria. Hacia un modelo de deporte escolar. Tesis Doctoral. Facultad de Ciencias de la Actividad Física y el Deporte. Universidad de Las Palmas de Gran Canaria.

ÁlAMO, J. M. (2004). El deporte escolar en Canarias. En Fraile (coord). El deporte escolar en el siglo XXI: Análisis y debate desde una perspectiva europea. (pp. 133-152). Barcelona: Graó.

ÁLAMO, J. M., AMADOR, F. y PINTOR, P. (2002). El deporte escolar: conquista de nuevos espacios en el mercado laboral. Revista Española de Educación Física y Deportes, 4, 5-10.

AUGUSTIN, J. P. (2003). Le sport et ses métiers. Paris: La Découverte.

BRYMAN, A. (2004). Social research methods. New York: Oxford University.

CAMPOS IZQUIERDO, A. (2007). Situación profesional de las personas que trabajan en funciones de actividad física y deporte en la Comunidad Autónoma Valenciana (2005). Valencia: Universidad de Valencia.

CAMPOS IZQUIERDO, A. y TOMÁS, X. (2005). La organización y estructura reglamentaria de los juegos deportivos municipales de Valencia. En Ajuntament de València (org.) I Congrés d'esport en edat escolar (pp. 30-40). Valencia: Ajuntament de València y Fundació Esportiva Municipal.

CAMY, J., CHANTELAT, P., y LE ROUX, N. (1999). Sport et emploi en Europe. France: Comisión Europeenne.

DELGADO, M. A. (2002). El deporte en los centros de enseñaza andaluces 2001. Excmo. Ayuntamiento de Dos Hermanas. Deporte y Municipio. II Congreso Nacio- 
nal del Deporte en Edad Escolar. (pp. 55-92). Patronato Municipal de Deportes. Sevilla: Excmo. Ayuntamiento de Dos Hermanas.

DÍAZ, R. (2005). El deporte extraescolar en Asturias. Deporte, ocio y competición. Análisis en una comarca. Lecturas: Educación Física y Deportes, 86. Disponible en: http://www.efdeportes.com.

FEDERACIÓN ESPAÑOLA DE MUNICIPIOS Y PROVINCIAS (1992). Manifiesto sobre el Deporte en la edad escolar. Madrid: FEMP.

FEU, S. (2002). Factores a tener en cuenta para una iniciación deportiva educativa: el contexto. Lecturas: Educación Física y Deportes, 51. Disponible en: http:// www.efdeportes.com.

FLINTOFF, A. (2003). The school sport co-ordinator programme: Changing the role of the physical education teacher? Sport, Education and Society, 8 (2), 231-250.

FRAILE, A. y DE DIEGO, R. (2006). Motivaciones de los escolares europeos para la práctica del deporte escolar. Un estudio realizado en España, Italia y Portugal. Revista internacional de sociología, 44, 85-109.

GARCÍA FERRANDO, M. (2002). La encuesta. En F. Alvira, M. García Ferrando y J. Ibáñez (Comps.), El análisis de la realidad social. Métodos y técnicas de investigación (ed. 3ㅜ) (pp. 141-170). Madrid: Alianza.

GIMÉNEZ, F. J. (2000). Fundamentos básicos de la iniciación deportiva en la escuela. Sevilla: Wanceulen.

GÓMEZ, J. (1997). Aspectos conceptuales y características del deporte escolar. Congreso del deporte en Euskadi. Santurce: Instituto Vasco de Educación Física.

GONZÁLEZ RIVERA, Maㅗ D. (2007). Elaboración y validación de una entrevista estandarizada por medio de cuestionario para el estudio del deporte escolar en los centros educativos de la Comunidad Autónoma de Madrid: la intervención didáctica y los recursos humanos en las actividades físico-deportivas extraescolares. Trabajo de investigación. Facultad de Ciencias de la Actividad Física y el Deporte: Universidad de Valencia.

GONZÁLEZ RIVERA, Maㅗ D. (2008). El deporte escolar en la Comunidad Autónoma de Madrid: intervención didáctica y recursos humanos en las actividades físicodeportivas extraescolares en los centros educativos. Tesis doctoral. Facultad de Ciencias de la Actividad Física y el Deporte: Universidad de Valencia.

GONZÁLEZ RIVERA, Maㅡ․ D. (Dir.) (2010). Estudio comparado sobre el mercado laboral y la intervención profesional de los técnicos deportivos en los programas de deporte escolar municipal. Madrid: Consejo Superior de Deportes.

GONZÁLEZ RIVERA, Maㅡㄹ. BLASCO, J. y CAMPOS IZQUIERDO, A. (2005). El deporte escolar en los centros educativos: aspectos metodológicos, formativos y laborales en las actividades físico-deportivas extraescolares desde la perspectiva del profesorado. En Ajuntament de València (Org.) I Congrés d'esport en edat escolar. Ajuntament de València y Fundació Esportiva Municipal: Valencia.

GONZÁLEZ RIVERA, Maㅡ D. y CAMPOS IZQUIERDO, A. (2010). La intervención didáctica del docente del deporte escolar, según su formación inicial. Revista de Psicodidáctica, 15(1), 101-120. 
GONZÁleZ, A. M. y OTERO, M. (2005). Actitudes de los padres ante la promoción de la actividad física y deportiva de las chicas en la edad escolar. Cuadernos de Psicología del Deporte, 5, 1-2.

GRIFFIN, L. L. y BUTLER, J. I. (2005). Teaching Games for Understanding: Theory, Research, and Practice. Champaign, IL: Human Kinetics.

HEINEMANN, K. (2003). Introducción a la metodología de la investigación empírica. Barcelona: Paidotribo.

HERNÁNDEZ, M. (Dir.) (2008). Deporte escolar: Estudio sobre la participación y puesta en marcha de programa de deporte escolar. Madrid: Consejo Superior de Deportes.

LUENGO, C. (2007). Actividad físico-deporttica extrescolar en alumnos de primaria. Revista Internacional de Medicina y Ciencias de la Actividad Física y el Deporte, 27. Disponible en: Http://cdeporte.rediris.es/revista/revista27/artactividadf41c.htm.

MACARRO, J. (2002). Estrategia para realizar actividades extraescolares: medio para desarrollar valores de convivencia en centros escolares. Espacio y tiempo. Revista de Educación Física, 35-36, 39-42.

MACAZAGA, A. M. (2004). El modelo de deporte escolar en el Plan Vasco de Deporte. En Fraile (coord.). El deporte escolar en el siglo XXI: Análisis y debate desde una perspectiva europea. Barcelona: Editorial Graó.

MARTÍN, J. M., CACHO, S. y MARTíN, C. (2001). Las actividades físicas y deportivas como actividades formativas y complementarias en la "Nueva Jornada Escolar", para los centros públicos en España. Lecturas: Educación Física y Deportes, 36. Disponible en: http://www.efdeportes.com.

MARTÍNEZ DEL CASTILLO, J. (Dir.) (1992). Proyección del mercado deportivo laboral en la España de los noventa. En los sectores de entrenamiento, docencia, animación y dirección. Madrid: CSD.

MITCHELL, S. A., OSLIN, J. L., y GRIFFIN, L. (2006). Teaching sport concepts and skills: A tactical games approach. Champaign, IL: Human Kinetics.

MOLLÁ, M. (2007). La influencia de las actividades extraescolares en los hábitos deportivos de los escolares. Revista Internacional de Medicina y Ciencias de la Actividad Física y el Deporte, 27. Disponible en: http://cdeporte.rediris.es/revista27/artinfluencia41f.htm.

MONTESINOS, J. M. (2005). El deporte en la edad escolar: Aspectos organizativos. En I Congrés d'Esport en edat escolar. Valencia: Ajuntament de València y Fundació Esportiva Municipal.

MORENO, J. A.; RODRÍGUEZ, P. L. y GUTIÉRREZ, M. (1997). Intereses y actitudes de los alumnos hacia la educación física. Premio Nacional de Artículos sobre Actividad Física y Deportiva, II Edición. Barcelona: COPLEFC.

ORTS, F. (2005). La gestión municipal del deporte en edad escolar. Barcelona: Inde.

ORTÚZAR, I. (2005). El deporte escolar y el profesorado de Educación Física. Tesis doctoral. Facultad de Ciencias Sociales y de la Comunicación. Universidad del País Vasco.

PARLAMENTO EUROPEO (2007). Informe sobre la función del deporte en la educación. Comisión de Cultura y Educación. 
PARKER, T. (2000). Professional developments in exercise and sport sciencie: An Australian perspective. ICSSPE Bulletin, 30, 12-13.

PETRY, K., FROBERG, K., y MADELLA, A. (2008). Higher Education in Sport in Europe: From labour market demand to training supply. Aaachen: Meyer \& Meyer.

PIÉRON, M. (2001). Participación de jóvenes europeos en actividades físicas y deportivas, actitudes hacia la escuela y la Educación Física. Consecuencias pedagógicas. En Manzón, V.; Sarabia, D.; Canales, F. C., Ruiz, F. y Torralba, R. (Coords.). Reflexiones y perspectivas de la enseñanza de la Educación Física y el Deporte Escolar en el nuevo milenio. Cantabria: A.D.E.F.

RIVADENEYRA, M. L. (2003). Las actividades físicas extraescolares como nexo entre la escuela y la vida cotidiana infantil: propuesta de una unidad didáctica. En I Congreso Europeo de Educación Física (pp. 354-362). Federación Internacional de Educación Física. Facultad de Ciencias del Deporte. Cáceres: Universidad de Extremadura.

SALVADOR, J. L. (1998). Evolución histórico-social del mercado laboral físicodeportivo. En M. González, R. Martín, J. L. Salvador y M. Vicente Pedraz (Eds.), Educación Física e deporte no seculo XXI, I (pp. 225-240). A Coruña: Universidade da Coruña.

SÁNCHEZ, J. M. y SÁNCHEZ, M. D. (2003). Deporte en edad escolar versus deporte escolar. En Ruiz, F. y González, E. P. Educación Física y deporte escolar. V Congreso Internacional de FEADEF. Valladolid: Europa Artes Gráficas.

SANTOS, M. (1998). La educación del ocio por medio de las actividades extraescolares. Actividades físicas extraescolares. Una propuesta alternativa. En Santos, M. y Sicilia, A. Actividades físicas extraescolares. (63-72). Barcelona: Inde.

SHIBLI, S., TAYLOR, P., NICHOLS, G., GRATTON, C., y KOKOLAKAKIS, T. (1999). The characteristics of volunteers in UK sport clubs. European Journal of Sports Management, Special Issue, 10-27.

TANI, G. (2007). Desporto e Escola. Que diálogo ainda é possível?. En J. Olímpio y J. M. Constantino (Coords.), Em Defesa do Desporto (pp. 269-287). Coimbra: Almedina.

TORRE, E. (1997). La actividad física-deportiva extraescolar y su interrelación con el área de Educación Física en el alumnado de enseñanzas medias. Tesis doctoral. Facultad de Ciencias de la Actividad Física y el Deporte. Universidad de Granada.

UREÑA, F. (2000). Deporte Escolar: Educación y Salud. En Ayuntamiento de Murcia (ed.). La educación olímpica. Murcia: Ayuntamiento de Murcia.

VELÁZQUEZ, R., DEVÍS, J., DE LA TORRE, E., CARRANZA, M. y FRAILE, A. (2004). Debate sobre algunos tópicos del deporte escolar en España. En Fraile (Coord.). El deporte escolar en el siglo XXI: Análisis y debate desde una perspectiva europea. Barcelona: Editorial Graó. 